\title{
Mineração de Dados no Domínio de Gestão de Recursos Naturais: Descoberta de estratégias em um RPG
}

\author{
Marla Pereira Melo ${ }^{1}$, Diana Francisca Adamatti ${ }^{1}$ \\ ${ }^{1}$ Programa de Pós-Graduação em Computação (PPGComp) \\ Centro de Ciências Computacionais (C3) \\ Universidade Federal do Rio Grande (FURG) - Rio Grande - RS - Brasil
}

\begin{abstract}
This paper presents the mapping of the players strategies in a roleplaying game, in the domain of natural resource management. The data collected (actions of the players in the game) were applied for automatic learning, through data mining. The decision trees generated by the WEKA software, obtained a percentage of correctness between $53 \%$ to $100 \%$, and through the analysis of the trees, the players' strategies were discovered. As results, 38 strategies were extracted. The mapped strategies help a better understanding of the game and provided the perception of how the players make their decisions.
\end{abstract}

Resumo. Este artigo apresenta o mapeamento das estratégias dos jogadores em um jogo de papéis, no domínio da gestão de recursos naturais. Os dados coletados (ações dos jogadores no jogo) foram aplicados no aprendizado automático, mediante a mineração de dados. As árvores de decisão geradas pelo software WEKA, obtiveram percentual de acerto entre 53\% a 100\%, e através da análise das árvores, as estratégias dos jogadores foram descobertas. Como resultados, conseguiu-se extrair 38 estratégias. As estratégias mapeadas auxiliam um melhor entendimento do jogo e proporcionaram a percepção de como os jogadores tomam suas decisões.

\section{Introdução}

A gestão dos recursos naturais compreende uma realidade complexa, pois envolve interações homens-ecossistemas: de um lado pensa-se em desenvolvimento socioeconômico e, de outro, em qualidade do meio ambiente. Estas questões socioambientais representam um grande desafio à humanidade em geral, mas com o auxílio de artefatos computacionais, pode ser possível criar métodos para auxiliar na busca de soluções.

No sentido dos estudos sobre os impactos ecológicos das ações humanas e questões de cooperação, resolução de conflitos no contexto da dinâmica dos sistemas socioambientais, a utilização dos sistemas multiagente representa uma das formas para construção de modelos a fim da obtenção de simulações das "inter-relações entre homens e ecossistemas" [Freire Vieira and Weber 1997], pois um sistema multiagente "equivale a reproduzir um mundo artificial que assemelha-se ao mundo observado" (Bousquet, 1995 apud [Freire Vieira and Weber 1997]).

A utilização integrada de Simulação Multiagente (MABS - Multi-Agent-Based Simulation) e Jogos de Papéis (RPG - Role-Playing Games), a qual consiste em uma técnica onde os jogadores "interpretam" uma personagem, criada dentro de um determinado cenário (ambiente) [Adamatti and Sichman 2007], iniciou-se com pesquisas desen- 
volvidas pelo CIRAD ${ }^{1}$ (Centre de coopération internationale en recherche agronomique pour le développement), França, no início dos anos 2000. Esse grupo desenvolveu uma metodologia denominada "ComMod: The Companion Modelling approach", onde os participantes têm papel decisivo no processo de tomada de decisão e entendimento dos problemas sócio-ambientais a serem resolvidos, sendo que inúmeros trabalhos já foram realizados em diversos países do mundo, incluindo o Brasil.

No sentido de observar o comportamento dos jogadores e suas tomadas de decisão no jogo, a técnica de mineração de dados se mostra eficiente, pois é uma técnica computacional utilizada para extrair informação útil, compreender melhor os dados, e criação de perfis [Tan et al. 2009]. Assim, este trabalho tem como objetivo o mapeamento de estratégias utilizadas pelos jogadores no RPG, no domínio da gestão de recursos naturais, através da aplicação da mineração de dados nos dados do RPG visando entender os perfis dos jogadores.

O trabalho está estruturado da seguinte forma: Na Seção 2 são apresentados os conceitos básicos de jogos de papéis e mineração de dados. Na Seção 3 é apresentada a metodologia aplicada para a realização deste trabalho. Na Seção 4 são apresentadas as estratégias mapeadas. Na Seção 5 são apresentadas as conclusões e trabalhos futuros.

\section{Revisão de Literatura}

\subsection{Jogos de papéis}

Os jogos de papéis, conhecidos como RPG (Role-Playing Games) "são um tipo de jogo onde os jogadores 'interpretam' uma personagem, criada dentro de um determinado cenário (ambiente) .... eles podem ser impressos (em mapas, fichas de personagens, etc.) eletrônicos ou oral" [Adamatti and Sichman 2007]."A forma tradicional de jogar RPG é conhecida pela comunidade como 'RPG de mesa', pelo fato de comumente ser jogado em torno de uma mesa com lápis, papel e dados" [Bittencourt and Giraffa 2003]. Nesta forma, são distribuídas fichas que contem a definição das ações de cada jogador [Luong et al. 2017]. As regras não limitam-se a personalidade de cada jogador, pois o jogador pode interpretar uma personagem bastante diferente de uma personalidade do mundo real. Assim, “os jogadores utilizam RPG como um 'laboratório social', isto é, como uma forma de experimentar uma variedade de possibilidades, sem sofrer as consequências do mundo real'"[Barreteau et al. 2003].

\subsection{Mineração de Dados}

Conforme [Tan et al. 2009], a mineração de dados é o processo de descoberta automática de informações úteis com o intuito de descobrir padrões e fornecer previsão do resultado de uma observação futura, isto é, ela é uma parte integral da descoberta de conhecimento em banco de dados (KDD - Knowledge Discovery in DataBases), que corresponde ao processo de transformação dos dados em informações úteis.

O processo de KDD, segundo [Tan et al. 2009], é dividido em três etapas:

- Pré-processamento: os dados brutos de entrada são transformados em formato apropriado à mineração de dados;

\footnotetext{
${ }^{1}$ www.cirad.fr

${ }^{2} \mathrm{https} / / /$ www.commod.org/en
} 
- Mineração de dados: escolha de métodos de descoberta de conhecimento, que combina métodos tradicionais de análise de dados com algoritmos sofisticados;

- Pós-processamento: análise dos resultados úteis, mediante a investigação dos dados e resultados da mineração de dados.

A mineração de dados através das árvores de decisão é utilizada neste trabalho. Em uma árvore de decisão, cada nodo folha recebe um rótulo de classe. Os nodos não terminais (nodo raiz e os nodos internos) contêm condições de testes de atributos para separar registros que possuam características diferentes [Tan et al. 2009], fazendo a comparação do valor de um atributo com uma constante e os nós da folha fornecem a classificação que se aplica a todas as instâncias que atingem a folha, "ou um conjunto de classificações ou uma distribuição de probabilidade em todas as classificações possíveis"[Frank and Hall 2011]. A classificação de uma instância desconhecida acontece de acordo com os valores dos atributos testados em nós sucessivos e, quando uma folha é alcançada, a instância é classificada de acordo com a classe atribuída à folha [Frank and Hall 2011].

\section{Metodologia}

Este trabalho faz parte do projeto Gestão participativa dos recursos hídricos utilizando jogos computacionais e sistemas multiagente ${ }^{3}$, que tem como objetivo principal a formalização e desenvolvimento de um jogo computacional para gestão participativa dos recursos hídricos no estado no Rio Grande do Sul, utilizando Simulação Multiagente e Jogos de Papéis. Na Figura 1 é apresentada a visão geral do projeto.

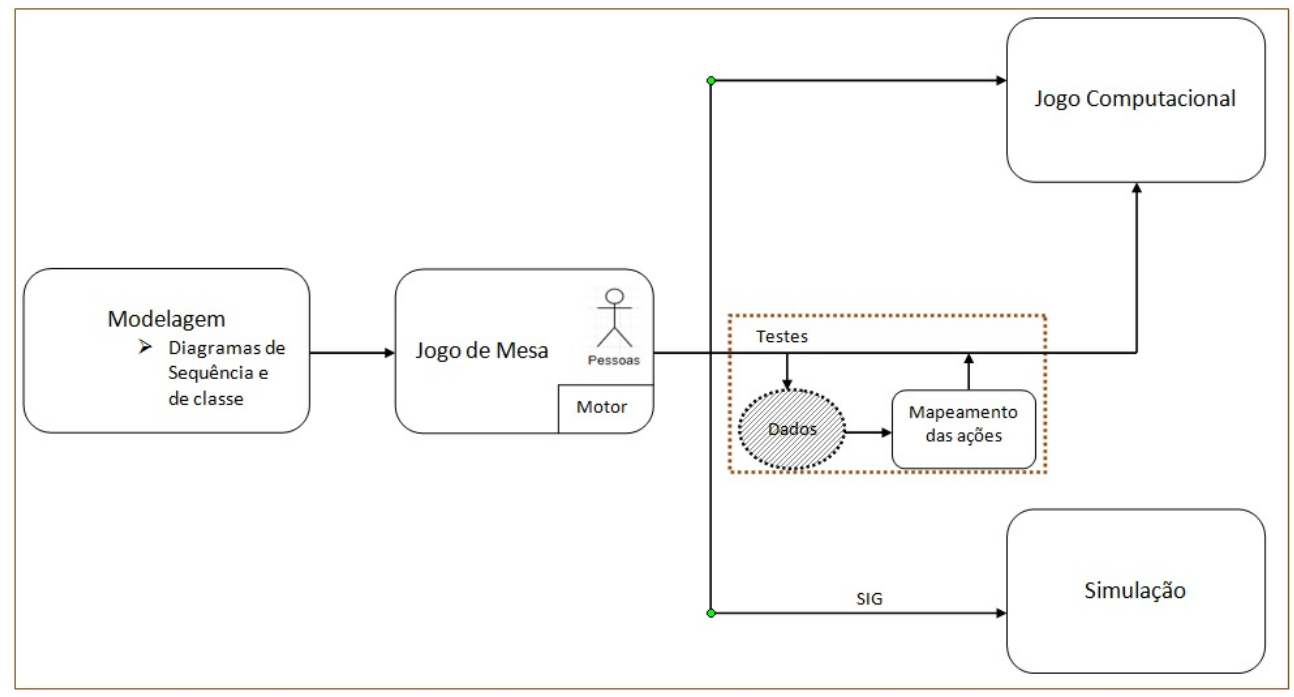

Figura 1. Visão geral do projeto

O jogo computacional e a simulação multiagente referem-se aos produtos finais do projeto. Entretanto, as fases da modelagem e do jogo de mesa são necessárias para atingir esse objetivo.

$\mathrm{Na}$ fase da modelagem ocorreram a definição e formalização das ações e interações dos papéis para o desenvolvimento das regras e do motor do jogo. O motor

\footnotetext{
${ }^{3}$ Projeto apoiado pelo Edital $\mathrm{N}^{\circ} 16 / 2017$
} 
do jogo é um programa na linguagem de programação Java desenvolvido por dois integrantes do projeto. Neste programa são computadas as ações escolhidas pelos jogadores no jogo de mesa ao final de cada rodada.

A etapa referente ao RPG de mesa, configura a base para o jogo computacional, e o enfoque deste presente trabalho pode ser visto na área destacada da figura, onde, os dados gerados a partir das ações escolhidas pelos jogadores nos testes do RPG de mesa representam a constituição de uma base de dados para a mineração de dados. Posteriormente, a estruturação do RPG de mesa será aplicada para o desenvolvimento do jogo computacional online e as estratégias descobertas por esse trabalho auxiliarão na construção do NPC (Non-Player Character).

Por fim, a etapa de simulação está relacionada a concepção do modelo de simulação dos dados reais provenientes do Sistema de Informações Geográficas (SIG).

Para a realização deste trabalho, todos os dados do RPG, correspondentes as variáveis do jogo e as ações possíveis de escolha, foram considerados para a mineração de dados, deste modo, o conjunto de dados para o treinamento apresenta 196 instâncias (linhas) e 87 atributos (colunas).

Desta forma, as etapas da metodologia deste trabalho foram:

1. Participação no processo de desenvolvimento do RPG de mesa, que inclui a criação das personagens, ações destas e fichas. A definição das personagens, ações e a estrutura do jogo RPG, foi realizada por um grupo de pesquisadores do projeto. O ambiente é representado por duas cidades, tendo como base a Bacia do canal de São Gonçalo e da Lagoa Mirim/RS. Existem seis tipos de personagens organizados em três grupos: reguladoras, fiscalizadoras e produtoras, como mostrado na Figura 2.

As personagens reguladoras são responsáveis por administrar os recursos financeiros, oriundos de impostos e taxações atrelados à sociedade, com o objetivo de controlar/mitigar a poluição (através da criação de leis, incentivos fiscais, obras para diminuir a poluição, etc.) sem prejudicar os mecanismos de produção. Neste ambiente, os agentes que assumem papéis de prefeito ou vereador pertencem ao grupo dos agentes reguladores, os quais podem negociar entre si para decidir quais ações realizar no ambiente.

Agentes fiscalizadores têm como objetivo fiscalizar ou informar irregularidades atreladas à produção e exploração do ambiente. Agentes fiscalizadores são aqueles que assumem os papéis de fiscal da agência ambiental (por exemplo, FEPAM no RS) ou ONG (Organização Não-Governamental). Ou seja, o fiscal é responsável por fiscalizar os agentes que pertencem ao grupo dos produtores, isto é, o fiscal pode, por exemplo, aplicar multas aos agentes produtores que forem pegos infringindo alguma lei/regra imposta pelos agentes reguladores. A ONG é responsável por informar aos agentes reguladores o estado atual dos níveis de poluição do ambiente, com o objetivo de conscientizar/pressionar os outros agentes a realizarem ações que diminuam os níveis de poluição.

Agentes produtores são responsáveis por explorar o ambiente com o objetivo principal de obter recursos financeiros. Estes agentes são os maiores geradores de poluição e, consequentemente, de recursos financeiros no ambiente, podendo assumir os papéis de empresário ou agricultor. O empresário é responsável 


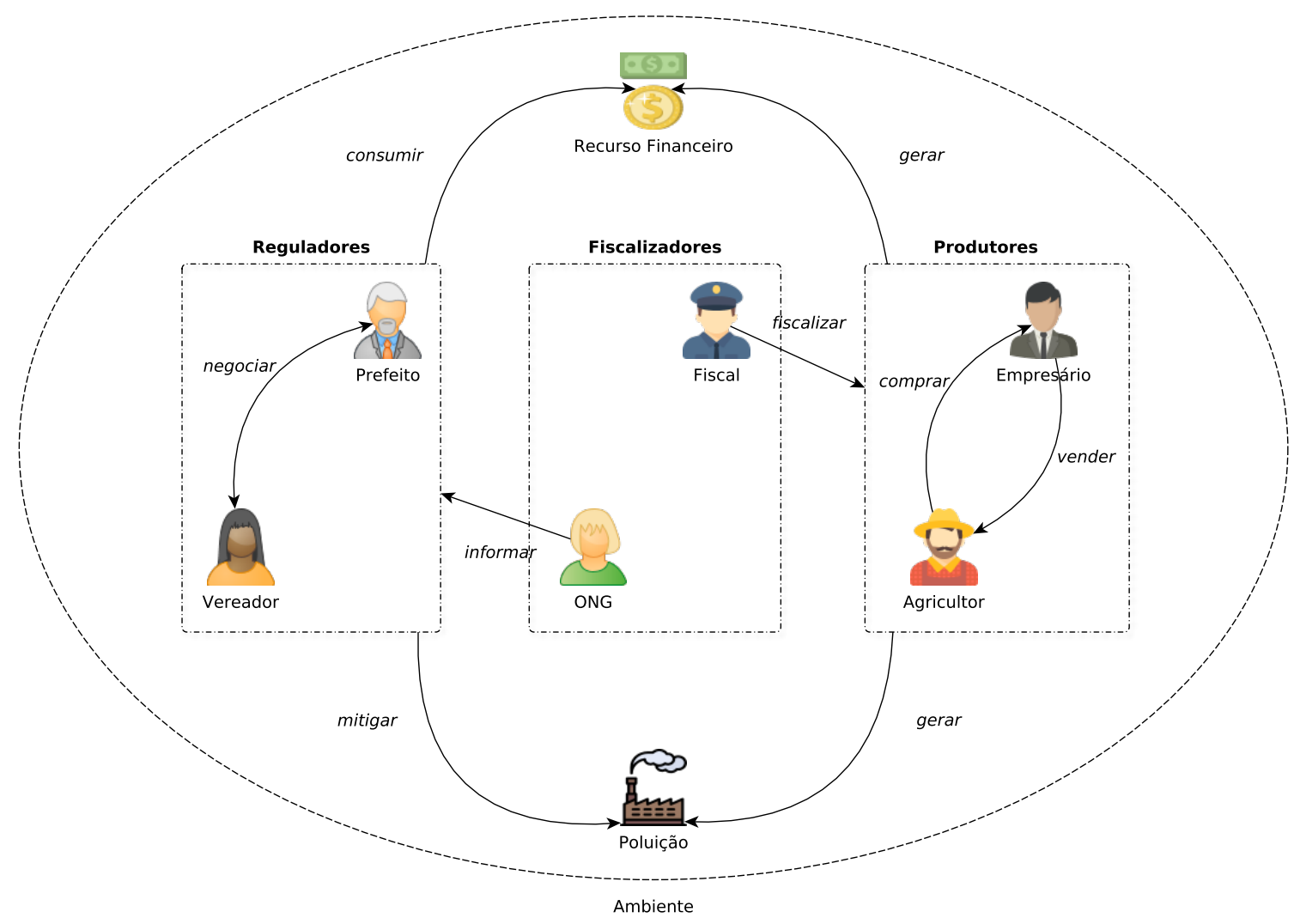

Figura 2. Diagrama de interação do RPG proposto [Leitzke et al. 2019].

por disponibilizar equipamentos e insumos necessários para a produção. No entanto, o agricultor é responsável por utilizar os equipamentos e insumos que julgar mais adequado para a sua produção. Deste modo, a interação entre os agentes produtores ocorre através da compra/aluguel e venda de equipamentos e insumos, onde um agente agricultor pode comprar de um agente empresário e, consequentemente, um empresário pode vender/alugar a um agricultor.

2. Modelagem de dados para a estruturação de base de dados utilizada neste trabalho, onde o conjunto de dados é formado pelas ações que os jogadores tomaram durante os testes do RPG de mesa, bem como pelos resultados dos cálculos do processamento das ações de cada jogador realizados pelo motor do jogo.

3. Processo de descoberta de conhecimento dos dados. Primeiro, os dados são transformados para o formato ARFF (Attribute-Relation File Format) para a mineração de dados no WEKA. Em seguida, cenários de testes são propostos e o software WEKA processa estes cenários. Os cenários foram construídos sob duas abordagens: de modo definido e de modo automático. A construção dos cenários de modo definido consistiu-se na escolha de diferentes conjuntos de atributos e classes preditivas para serem testadas, a partir de ações possíveis para os jogadores; e a construção de modo automático constituiu-se por uma seleção de atributos automática, com o objetivo de construir cenários de forma totalmente automática.

4. Análise dos cenários de testes propostos obtidos a partir da terceira etapa.

Este trabalho aborda as árvores de decisão para a tarefa de aprendizado. No software WEKA, foi utilizado o classificador J48 que implementa o algoritmo C4.5 para a 
construção de árvores de decisão. Este algoritmo deriva da técnica de divisão e conquista e aprende a partir de instâncias do conjunto de treinamento [Witten and Frank 2002]. A cada nó, o algoritmo escolhe um atributo mais eficientemente e subdivide o conjunto das amostras em subconjuntos homogêneos e caracterizados por sua classe. O critério é o ganho de informação obtida na escolha do atributo para subdivisão (Quilan, 1993; Hall et al., 2009) apud [Giasson et al. 2013]. O atributo que possui o maior ganho de informação representa o parâmetro que influencia a estratégia dos jogadores, ou seja, os nodos não terminais da árvore.

\section{Resultados}

Nesta seção são apresentadas as estratégias dos jogadores encontradas por meio da interpretação de todas as árvores de decisão obtidas pela classificação, no software WEKA $\mathrm{Weka}^{4}$, onde utilizou-se classificador $\mathrm{J} 48$ com a opção de teste: conjunto de treinamento, para todos os testes. Com a análise das árvores de decisão geradas, propõe-se extrair as estratégias dos jogadores que interpretaram os papéis de agricultores, empresários, fiscais e prefeitos. O papel vereador para essa análise não foi considerado, pois essa personagem gerou poucas ações no jogo, o que inviabilizou sua análise neste trabalho. O papel ONG não gera ações, pois apenas informa situações que podem ocorrem durante o jogo.

Na Tabela 1 são apresentados os resultados obtidos pelos cenários.

Tabela 1. Resultados

\begin{tabular}{l|l|l|l}
\hline & Cen. Definidos & Cen. Automáticos & Total \\
\hline Cenários & 22 & 48 & 70 \\
\hline Árvores geradas & 22 & 44 & 66 \\
\hline Árvores repetidas & 1 & 6 & 7 \\
\hline Árvores analisadas & 21 & 38 & 59 \\
\hline Estratégias & 14 & 30 & 44 \\
\hline
\end{tabular}

Nas Tabelas 2 e 3 são apresentadas algumas das estratégias mapeadas, após a análise de todas as árvores de decisão, bem como os papéis que assumem as estratégias.

Tabela 2. Mapeamento das estratégias dos jogadores em cenários de teste definidos

\begin{tabular}{l|l}
\hline Estratégia & Papel \\
\hline 1. Tentar ter alta produtividade não importando a poluição. & Agricultor \\
\hline $\begin{array}{l}\text { 2. Agricultores e empresários que têm baixa produtividade e baixa } \\
\text { poluição realizam mais transferências de recursos em comparação aos } \\
\text { agricultores e empresários com alta produtividade e alta poluição. }\end{array}$ & $\begin{array}{l}\text { Agricultor } \\
\text { Empresário }\end{array}$ \\
\hline $\begin{array}{l}\text { 3. Fiscais ignoram alguns casos e não multam agricultores que geram } \\
\text { alta poluição. }\end{array}$ & Fiscal \\
\hline
\end{tabular}

\footnotetext{
${ }^{4}$ https://www.cs.waikato.ac.nz/ml/weka/
} 
Os exemplos, demostrados das estratégias mapeadas dos papéis agricultores, empresários e fiscais, mostram que os jogadores tem intenção de lucrar, buscam cooperar, assim como negociar.

Tabela 3. Mapeamento das estratégias dos jogadores em cenários automáticos

\begin{tabular}{l|l}
\hline Estratégia & Papel \\
\hline $\begin{array}{l}\text { 4. Agricultores buscam o equilíbrio entre plantações que geram } \\
\text { poluição e plantação que gera um mínimo de poluição. }\end{array}$ & Agricultor \\
\hline $\begin{array}{l}\text { 5. Quando o valor da negociação dos preços de venda dos agrotóxicos } \\
\text { é normal e alto, o número de agricultores que utilizam agrotóxico dimi- } \\
\text { nui. }\end{array}$ & $\begin{array}{l}\text { Agricultor } \\
\text { Empresário }\end{array}$ \\
\hline $\begin{array}{l}\text { 6. Os prefeitos que realizam tratamento de esgoto também realizam o } \\
\text { tratamento de água. }\end{array}$ & Prefeito \\
\hline
\end{tabular}

Em geral, a maioria das estratégias mapeadas são referentes ao papel do agricultor, isso porque eles possuem mais ações no jogo.

A estratégia para o papel agricultor mapeada: 1. Tentar ter alta produtividade não importando a poluição é observada na árvore de decisão onde há agricultores que tiveram alta produtividade de '2445'e os que tiveram produtividade de '1760', e geraram o mesmo alto nível de poluição, mostrado na Figura 3, respectivamente, nos casos $3^{\circ}$ e $1^{\circ}$, mas há também os agricultores que tiveram produtividade de '2245' e geraram menor poluição, indicando a possibilidade de ter alta produtividade e poluir menos. Dessa forma, os agricultores que procuraram obter uma produtividade alta, além de não conseguir, geraram poluição como os agricultores que produziram mais. Na Figura 3 é destacada a estratégia extraída.

A estratégia para os papéis agricultores e empresários extraída: 2. Agricultores $e$ empresários que têm baixa produtividade e baixa poluição realizam mais transferências de recursos em comparação aos agricultores e empresários com alta produtividade e alta poluição. É demostrada quando observa-se na árvore de decisão que os agricultores e empresários que tiveram alta produtividade, isso resulta em ganhos altos de recursos, não realizaram transferência de recurso. Já os que tiveram produtividade baixa, com isso baixo ganhos de recursos realizam transferências de recursos.

A estratégia para o papel fiscal extraída: 3. Fiscais ignoram alguns casos e não multam agricultores que geram alta poluição, onde ao observar o fiscal, este aplica multa diferentes para agricultores que geraram o mesmo nível de poluição, assim como fiscais que não aplicaram multa para agricultores que deveriam ser multados.

A estratégia para o papel agricultor extraída: 4. Agricultores buscam o equilíbrio entre plantações que geram poluição e plantação que gera um mínimo de poluição. Essa estratégia indica que alguns agricultores buscam um equilíbrio para tentar diminuir a poluição gerada por suas plantações, plantando uma semente que polui mais e uma que polui menos.

A estratégia para os papéis agricultor e empresário extraída: 5. Quando o valor da negociação dos preços de venda dos agrotóxicos é normal e alto, o número de agricultores que utilizam agrotóxico diminui. Para os agricultores, a compra de agrotóxico é 


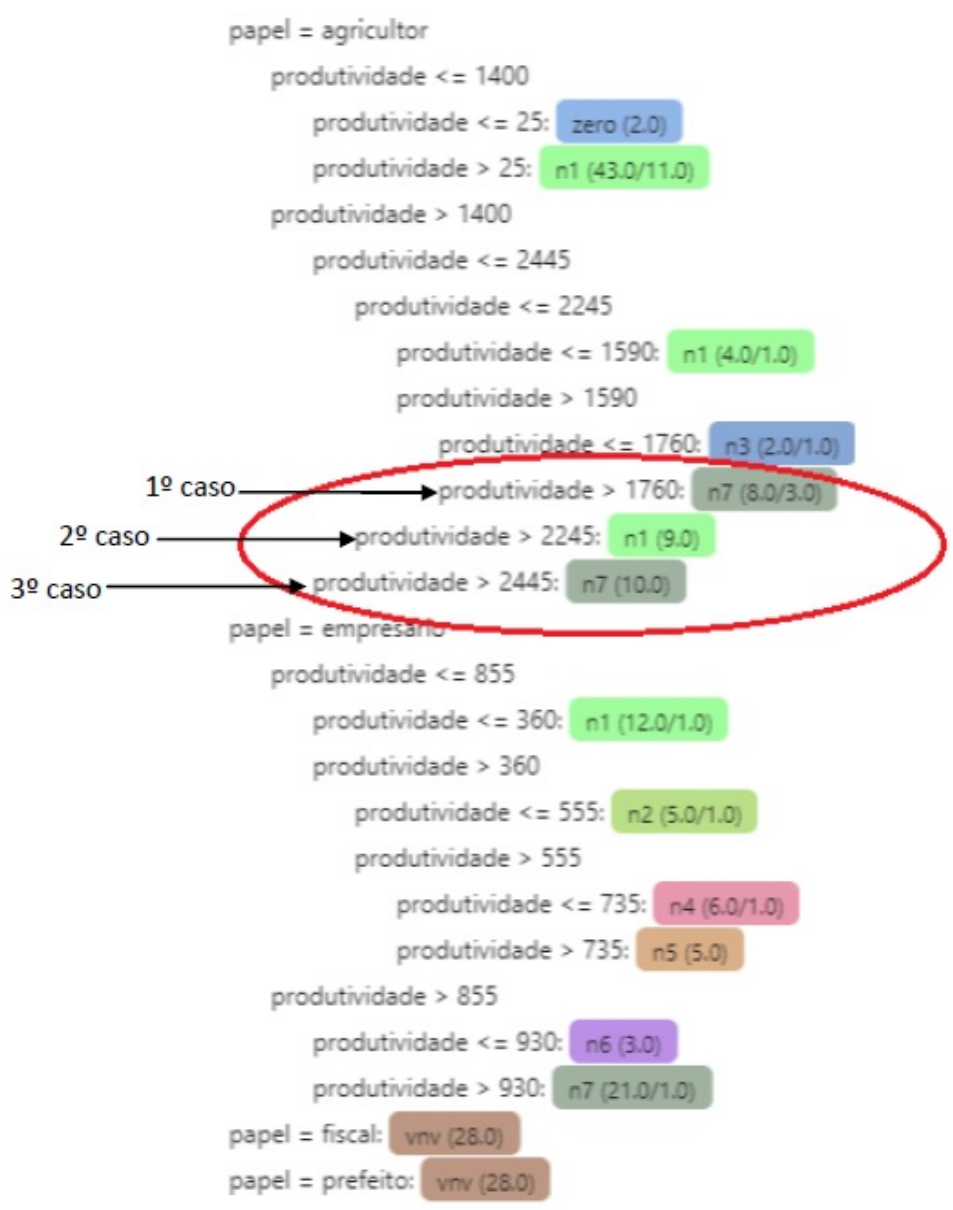

Figura 3. Estratégia encontrada por cenário definido para o papel de agricultor

interessante se o empresário o vende pelo preço baixo.

A estratégia para o papel agricultor extraída: 6. Os prefeitos que realizam tratamento de esgoto também realizam o tratamento de água ficou evidente na árvore de decisão, o tratamento de água e esgoto são medidas para diminuição da poluição. Na Figura 4 é apresentada a estratégia extraída.

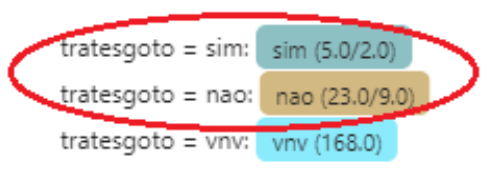

Figura 4. Estratégia encontrada por cenário automático para o papel de prefeito

\section{Conclusões e Trabalhos Futuros}

Este artigo apresentou o mapeamento das estratégias dos jogadores, para obter os perfis dos papéis no RPG de mesa, através do aprendizado de máquina. Foi proposta a construção de diversos cenários, a fim de aplicar a técnica de mineração de dados às ações escolhidas pelos jogadores do RPG. Os testes realizados ocorreram com o conjunto de treinamento formado pelos dados do RPG de mesa. 
Dessa forma, foi possível obter um mapeamento de 44 estratégias utilizadas pelos jogadores, por meio das observações das árvores de decisão. Por meio da construção 22 cenários baseados nas ações dos jogadores extraiu-se 14 estratégias. Já pela construção automática, dos 44 cenários, 30 foram extraídas.

Ainda, observa-se que os jogadores conseguem entender melhor o jogo depois de jogarem algumas rodadas e com isso, eles conseguem traçar melhores estratégias para talvez aumentar sua produtividade e/ou diminuir a poluição, já que são diversos os objetivo que o jogador pode definir durante o jogo, ao interpretar um papel. Além disso, a personalidade individual dos jogadores e a dinâmica do grupo, no jogo, podem influenciar nas ações tomadas.

Dada a complexidade da dinâmica do RPG, como trabalhos futuros, vislumbra-se:

- Realizar o registro dos diálogos (via vídeos, por exemplo), podendo ser útil para efetuar aplicar aprendizado automático sobre esses dados. Durante os testes do RPG de mesa, observou-se que houveram muitas iterações como planejamento e acordos entre os jogadores.

- Utilizar outras técnicas de mineração de dados, como Random Tree e Random Forest, para descobrir outras estratégias na base da dados obtida pelos jogos RPG de mesa.

- Utilizar PCA (Principal Component Analysis) para definir os atributos mais relevantes e depois reaplicar J-48, e outras técnicas de mineração de dados, também com o intuito de descobrir novas estratégias nos dados dos jogos RPG de mesa.

\section{Agradecimentos}

O presente trabalho foi realizado com apoio da Coordenação de Aperfeiçoamento de Pessoal de Nível Superior - Brasil (CAPES) - Edital N 16/2017.

\section{Referências}

Adamatti, D. F. and Sichman, J. S. (2007). Inserçao de Jogadores Virtuais em Jogos de Papéis para Uso em Sistemas de Apoio a Decisao em Grupo: um Experimento no Domınio da Gestao de Recursos Naturais. PhD thesis, $\mathrm{PhD}$ thesis, Universidade de Sao Paulo, Sao Paulo, Brasil.

Barreteau, O., Le Page, C., and D'aquino, P. (2003). Role-playing games, models and negotiation processes. Journal of Artificial Societies and Social Simulation, 6(2).

Bittencourt, J. R. and Giraffa, L. M. (2003). Modelando ambientes de aprendizagem virtuais utilizando role-playing games. In Brazilian Symposium on Computers in Education (Simpósio Brasileiro de Informática na Educação-SBIE), volume 1, pages 683-692.

Frank, E. and Hall, M. A. (2011). Data mining: practical machine learning tools and techniques. Morgan Kaufmann.

Freire Vieira, P. and Weber, J. (1997). Gestão de recursos naturais renováveis e desenvolvimento: novos desafios para a pesquisa ambiental. Cortez Editora.

Giasson, E., Hartemink, A. E., Tornquist, C. G., Teske, R., and Bagatini, T. (2013). Avaliação de cinco algoritmos de árvores de decisão e três tipos de modelos digitais de elevação para mapeamento digital de solos a nível semidetalhado na bacia do lageado grande, rs, brasil. Ciência Rural, 43(11):1967-1973. 
Leitzke, B., Farias, G., Melo, M., Gonçalves, M., Born, M., Rodrigues, P., Martins, V., Barbosa, R., Aguiar, M., and Adamatti, D. F. (2019). Sistema multiagente para gestão de recursos hídricos: Modelagem da bacia do são gonçalo e da lagoa mirim.

Luong, B., Thangarajah, J., and Zambetta, F. (2017). A bdi game master agent for computer role-playing games. Computers in Entertainment (CIE), 15(1):4.

Tan, P.-N., Steinbach, M., and Kumar, V. (2009). Introdução ao datamining: mineração de dados. Ciência Moderna.

Witten, I. H. and Frank, E. (2002). Data mining: practical machine learning tools and techniques with java implementations. Acm Sigmod Record, 31(1):76-77. 